\title{
Utilización de GPU-CUDA en el Procesamiento Digital de Imágenes
}

Francisco Javier Enríquez Aguilera

Jesús Martín Silva Aceves

Soledad Vianey Torres Argüelles

Erwin Adán Martínez Gómez

Gabriel Bravo Martínez

Instituto de Ingeniería y Tecnología

Universidad Autónoma de Ciudad Juárez

\section{RESUMEN}

EL procesamiento de imágenes es una herramienta de gran utilidad en diversas aplicaciones como video vigilancia, reconstrucción de imágenes, información geográfica y médica. Sin embargo, estas aplicaciones requieren una gran demanda computacional para ser llevadas a cabo en el menor tiempo posible, aún y que se desarrollan nuevos algoritmos, suelen ser restrictivos para implementarse en tiempo real en sistemas que solo se basan en CPU. Afortunadamente, estos algoritmos pueden ser analizados para llevarse a cabo en plataformas de cómputo paralelo, como las GPU-CUDA. En este trabajo se analizan diferentes revistas de la IEEE desde el 2013, donde se paralelizaron algoritmos con estas aplicaciones y se implementaron con ayuda de GPU's. Se destaca que el uso de esta herramienta ha ido en crecimiento en el ámbito científico, en diferentes ramas de la ciencia.

Palabras clave: CPU, GPU, CUDA, Procesamiento Digital de Imágenes, Nvidia.

\section{Introducción}

En 1965 Gordon Moore predijo que el número de transistores en un circuito integrado se iba a duplicar cada año, a esto se le nombro la "ley de Moore" (Moore, 1998), más tarde en 1975 el mismo Moore cambiaria esa predicción mencionando que la duplicación sería cada dos años (Moore, 2005) y que existía un límite en el número de transistores a integrar en el periodo entre el 2015 y 2020.

Dicho tope conlleva a su vez a una limitación en el tiempo de velocidad de procesamiento de los sistemas de programación secuencial basados en Unidad de Procesamiento Central (CPU) y ha ocasionado que actualmente se utilicen procesadores multinúcleo y plataformas de computación paralelo (Vogel, 2013).

Estas plataformas incluyen hardware y software de programación necesarios, lo que permite computar con más de un procesador de manera simultánea. Esto ha generado la necesidad de utilizar las ventajas de la programación de cómputo paralelo (Marcellino and Navarra, 2016), aplicado a plataformas paralelas.

Existen diferentes plataformas como las basadas en un Arreglo de Compuertas 
Programable en Campo de muy alta velocidad (FPGA) , las basadas en procesadores multinúcleo utilizando lenguajes especializados como OpenMP (Tagliavini, Cesarini and Marongiu, 2018) y las basadas en Unidades de Procesamiento Gráfico (GPU).

El procesamiento de imágenes es una de las clases de algoritmos más básicos y omnipresentes, sin embargo, plantea un desafío la gran demanda de datos que tiene el entorno en que están integrados (Wu et al., 2016).

Entre las plataformas paralelas existentes destaca la Arquitectura Unificada de Dispositivos de Cómputo (CUDA) (Saxena, Sharma and Sharma, 2014), (Vokorokos et al., 2014), la cual permite acelerar aplicaciones de cómputo, aprovechando el poder de las GPU's (NVIDIA, no date). Recientemente se ha incrementado el uso de la plataforma CUDA (Pawar, 2017), Sin embargo, solo se detallan revisiones del estado del arte llevadas a cabo para acelerar la velocidad de procesamiento digital de imágenes (PDI) en ciertas aplicaciones como la médica (Weinlich et al., 2013)(Jiansen Li et al., 2014)(Lee et al., 2013) y no cubre las diferentes aplicaciones de procesamiento de video utilizando GPU en video vigilancia (Jha and Trivedi, 2013),
(Devani, Nikam and Meshram, 2015), (Deligiannidis and Arabnia, 2014), procesamiento de imágenes de Radar de Apertura Sintética (SAR) (Fatica and Phillips, 2014), mejora de súper resolución de imágenes (Feng, Zhang and Gao, 2015), reconocimiento de objetos utilizando descriptor de Fourier (Haythem et al., 2014), criptografía, seguimiento de objetos, reducción de ruido (Yazdanpanah et al., 2014), reconstrucción de imágenes (Zhu et al., 2013)(Kau and Chen, 2013)(Heidari, 2013), detección de rostros (Sun et al., 2013), modelos de actuadores planares (Xu, Dinavahi and $\mathrm{Xu}, 2016)$, etc.

Por lo que una revisión del estado del arte que cubra todas estas aplicaciones de procesamiento digital de imágenes sirve como una visión general e introducción a las implementaciones de GPU más relevantes existentes en la actualidad. En este trabajo se analiza el uso de la plataforma CUDA en el campo del procesamiento de imágenes en sus diferentes áreas en la que se ha utilizado en Revistas del IEEE desde el 2003. Se analizan las contribuciones con la paralelización de algoritmos que son restrictivos por el tiempo que necesitan para procesarse mediante arquitecturas secuenciales y propuestas para mejorar el uso de este tipo de plataformas.

\section{USO DE GPU EN EL PROESAMIENTO DE IMÁGENES}

Las empresas de imagenología y video juegos tienen un rol impresionante en el desarrollo de hardware gráfico. Desde 1999 Nvidia y ATI (las dos principales compañías de desarrollo de tarjetas gráficas), han tenido una curva incremental de producción de tarjetas (Haythem et al., 2013). Con el nacimiento especializado del software para las arquitecturas paralelas GPU's en el 2012 se detonó el uso de estas plataformas para resolver problemas científicos, por ejemplo, física (Duchateau et al., 2017), matemáticas (Marcellino and Navarra, 2016), química (Phusomsai and So-in, 2016) y biología (Phusomsai and So-in, 2016), (Jia, Ziegenhein and Jiang, 2014). Existen diferentes disciplinas en las que actualmente se utilizan las GPU's para resolver diversas problemáticas, en la figura 1 se muestras las diferentes aplicaciones en las que se utiliza desde el 2009, así como la cantidad de artículos de revista en las diferentes revistas 
que se encontraron, esta información es analizada por Clarivate Analytics del Web of Science.

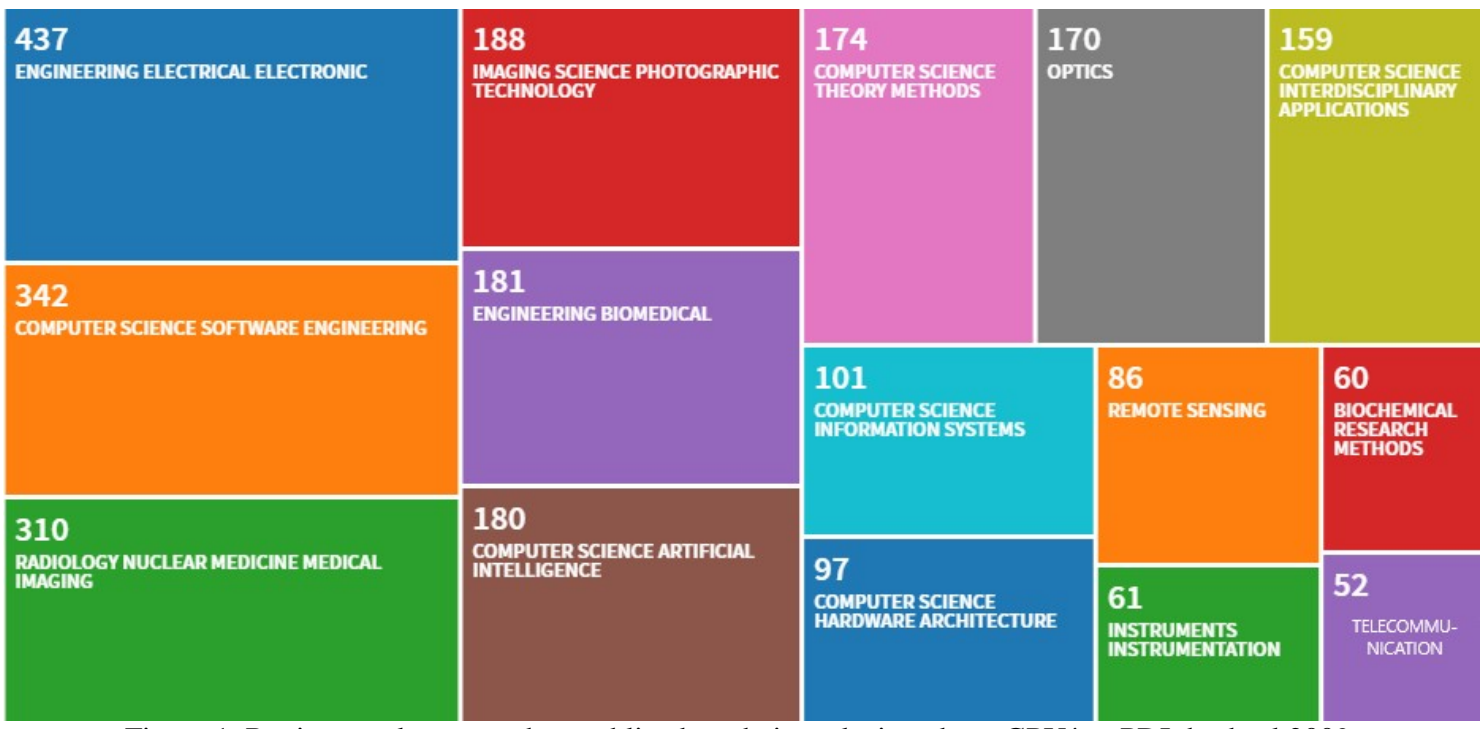

Figura 1. Revistas en las que se han publicado trabajos relacionados a GPU’s y PDI desde el 2009.
Cabe resaltar que el PDI en aplicaciones médicas son las que tienen mayor cantidad de artículos publicados en diferentes revistas, sin embargo, existen otras aplicaciones como Instrumentación, telecomunicaciones, videovigilancia, etc., que también tienen la necesidad de acelerar el tiempo de procesamiento de imágenes utilizando GPU.

En 2008, el tipo de documento "proceedings paper" (PP) fue asignado en la base de datos Web of Science para artículos de revista los cuales fueron inicialmente presentados en conferencias y posteriormente adaptados para publicaciones en un revista (González-Albo and Bordons,
2011). Se observa en los análisis del Clarivate Analytics que existes dos diferentes tipos de publicaciones: 1723 artículos de revista (Journal en inglés) y 125 PP o artículos de procedimientos.

Además, en la figura 2 se muestran la cantidad de publicaciones por país (con más de 50 publicaciones y desde el 2010), utilizando las GPU's para el PDI, se observa que los Estados Unidos de América y la República Popular de China lideran la cantidad de artículos, siendo seguidos por los países de Alemania, España, Corea del Sur, Francia, Canadá, Italia, Inglaterra y Japón. 


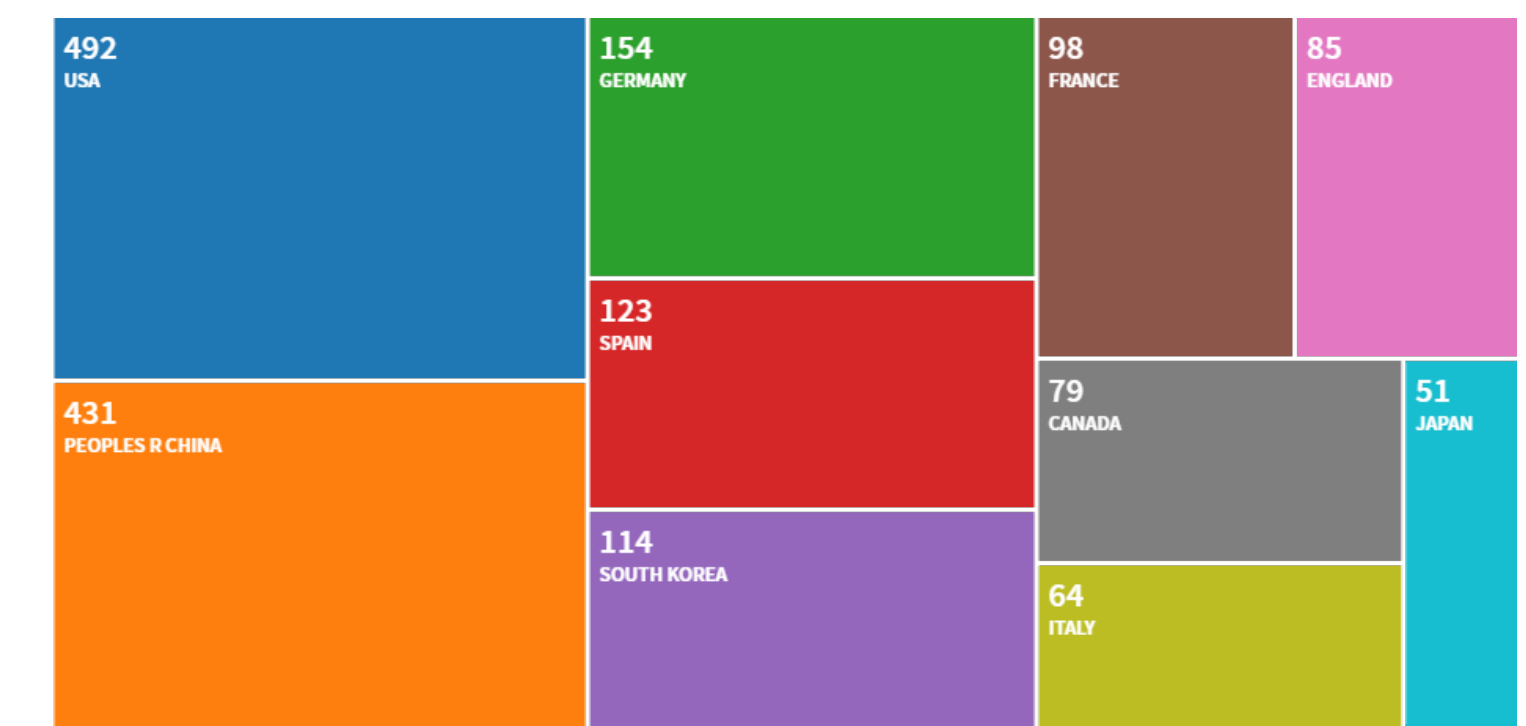

Figura 2. Publicaciones por país en aplicaciones basadas en GPU’s y PDI desde el 2009 (países con más de 50 publicaciones)

\section{Video vigilancia}

Chistoper Thorpe, et al. introducen un esquema de encriptación espacial al difuminar de manera estratégica los contenidos de una imagen/video de video vigilancia, para lograr obtener una velocidad de procesamiento en tiempo real, se logró procesar alrededor de 20 cuadros por segundo (fps), utilizando una GPU CUDA GTX460, en imágenes de 640 x 480 pixeles (Loock et al., 2014).

La captura de movimiento de un cuerpo es de gran utilidad en la video vigilancia, en (Zhang et al., 2013) se propone una aproximación para el seguimiento de cuerpos 3D en tiempo real, utilizando varias cámaras de video para evitar la oclusión; se utilizaron 8 cámaras y se obtuvo un máximo de velocidad de 9fps.

Otro trabajo para la reconstrucción 3D del movimiento de objetos, utilizando varias cámaras, se presenta en (Alexiadis, Zarpalas and Daras, 2013), utilizando una tarjeta NVidia GTX 560Ti se logró procesar a una velocidad de 10fps. Mientras que en (Zhou, Li and $\mathrm{Hu}, 2016$ ) se propone el seguimiento de objetos discriminando la función de velocidad del mismo, para obtener una mejor evolución del contorno (Zhou, Li and $\mathrm{Hu}, 2016$ ).

El uso de imágenes tipo estéreo (imágenes tomadas desde un par de cámaras), es de gran utilidad para evitar oclusiones, en las imágenes obtenidas, pero requieren de emparejamiento de las imágenes obtenidas, mediante métodos de evaluación computacional, por lo que en (Kowalczuk, Psota and Perez, 2013), se presenta este método utilizando CUDA y una NVidia GTX 580 para poder llevar a cabo el procesamiento en tiempo real (62fps).

El análisis de características de una imagen puede considerarse como una aplicación de video vigilancia, ya que con esto se puede lograr el reconocimiento de objetos (Bahri et al., 2017), como las huellas digitales, la seguridad, por lo que Pablo Gutiérrez, et al. proponen un sistema para análisis de concordancia de huellas digitales de alto rendimiento para bases de datos de gran tamaño basado en GPU, se logró obtener una mejora de $100.8 \mathrm{x}$ comparado 
con un procesamiento basado en CPU (Gutierrez et al., 2014).

La extracción en primer plano es un componente importante en aplicaciones multimedia, cuando se segmenta el objeto del primer plano de una imagen de fondo de una imagen $o$ video se pude realizar el reconocimiento de objetos (que puede utilizarse con el fin de video vigilancia), edición de imágenes/video, y compresión (Zhao, Zhang and Fang, 2015); Mengyao Zhao, et al. presentan un método de extracción en tiempo real de este componente, mediante el uso de una GPU, obteniendo una 30.3fps en promedio (Zhao et al., 2015).

El uso de redes y gráficos a gran escala son aplicaciones de datos intensivos que tienen aplicación en el monitoreo de tráfico y redes, ingeniería de software, bioinformática y telecomunicaciones; en (Zwan, Codreanu and Telea, 2016) se demuestra que el uso de GPU's para este tipo de procesamiento puede ayudar a acelerar hasta 50x el tiempo de procesamiento, comparado con las implementaciones en CPU.

El estudio de consumo de energía para la paralelización de un algoritmo de reconocimiento de Iris utilizando GPU es publicado en (Rakvic, Broussard and Ngo, 2016), donde se demuestra que el uso eficiente de los algoritmos puede disminuir el consumo de energía de una GPU. Un método acelerado del registro de imágenes basado en funciones sin rotación es presentado en ( $\mathrm{Yu}$ et al., 2015), el registro de imágenes es un factor clave en diversas aplicaciones, como reconocimiento de patrones aplicados a la video vigilancia o procesamiento de imágenes médicas.

La detección y seguimiento de objetivos aéreos utilizan la firma infrarroja del objeto aéreo, debido a la reflexión de la radiación del sol en el objeto, el tiempo para realizar este tipo de procesamiento tiene un costo de procesamiento elevado, por lo que en (Guo et al., 2016), se propone una comparación de implementaciones utilizando OpenMP, OpenACC y CUDA para este tipo de procesamiento, los resultados arrojaron que las mejoras que se obtuvieron fueron de $15 x, 140 x$ y $426 x$ respectivamente.

Una aplicación de video vigilancia en autos, se propone en ( $\mathrm{Li}$, Mogelmose and Trivedi, 2016), con la finalidad de reconocer señales de tráfico mediante redes neuronales convolucionales. Aunque la captura de movimiento es utilizada en aplicaciones como animación computarizada y sistemas de tele-inmersión, también se pueden utilizar para activar alarmas, en (Shuai et al., 2017) se propone la detección de esqueletos utilizando múltiples cámaras y GPU-CUDA logrando una mejor robustez en la propuesta. Alexander Filonenlo, et al. proponen un sistema de video vigilancia rápido para detección de humo utilizando CUDA, este método toma la ventaja del uso de cámaras de video vigilancia, de donde obtienen la información necesaria para la detección de humo, se logró mejorar el tiempo de detección a lo planteado en la literatura, tanto con el solo uso de CPU y todavía más con el sistema basado en CPU-GPU (Sun and Wang, 2015).

\section{Reconstrucción de Imágenes}

El desentrelazado de imágenes, se utiliza la transmisión de los sistemas de televisión digital, el rendimiento del entrelazado y desentrelazado es un factor clave para la calidad de las imágenes que se mostrará en el receptor, en (Method et al., 2014) se 
propone un método paralelo eficiente basado en GPU, que es adaptativo para el desentrelazado en campo con interpolación de los pixeles perdidos, la arquitectura propuesta generó una mejora de velocidad de 94.6x comparado con el método basado en CPU.

El mapeado de texturas es una herramienta que sirve para incrementar el realismo y fidelidad de imágenes, en las mesoestructuras se presentan deformaciones geométricas en imágenes $3 \mathrm{D}$ y pueden ser reemplazadas por operaciones de imágenes bidimensionales mediante el uso de GPU, como se plantea en (Nykl, Mourning and Chelberg, 2014). Pablo Enfedaque, et al. presentan la implementación basada en registros, de la Transformada Wavelet Discreta (DWT), mediante el uso de una GPU, sus resultados obtuvieron una mejora de velocidad de cuatro veces comparado con las implementaciones en GPU encontrados en la literatura antes de diciembre del 2015 (Enfedaque, Auli-Llinas and Moure, 2015).

La compresión de imágenes tiene como finalidad representar la información en el menor número de bits posible, pero de igual o mayor importancia es la descompresión o recuperación de la información original en aplicaciones como teleconferencia, cinema digital, etc. en (Enfedaque, Auli-Llinas and Moure, 2017) se propone el uso de GPU para mejorar los tiempos de procesamiento en estas aplicaciones, logrando obtener hasta 30x de mejora para aplicaciones de alta resolución $(4 \mathrm{~K})$.

En la visualización interactiva el cuello de botella ya no es el tiempo de cómputo, sino más bien el de transmisión de la información, por lo que en (Grosset et al., 2017) se publica un algoritmo de composición de imágenes, que funciona tanto en supercomputadoras basadas en CPU y GPU, y se enfoca en evitar la superposición en las comunicaciones de los datos, con la finalidad de equilibrar la carga de trabajo.

El filtro de la mediana es una técnica para suavizar y remover ruido en imágenes, varios de las implementaciones paralelas utilizan algoritmos de ordenamiento, para reacomodar los valores dentro de una ventana de filtrado y así calcular la mediana de los valores ordenados; esto produce un cuello de botella, al utilizar algoritmos secuenciales para este procesamiento, en (Green, 2018), se propone un método basado en histograma y GPU-CUDA, que no requiere el ordenamiento de los datos. La interpolación es otra técnica utilizada para la reconstrucción de imágenes de ultra alta definición (HD), en (Optimization, 2018), se propone la optimización paralela usando GPU de un modelo de interpolación auto regresiva en imágenes, el algoritmo propuesto obtiene mejoras de $147.3 x$ para la imagen Lena y 174.8x para video a 720p.

\section{Información Geográfica}

En aplicaciones de información geográfica es de gran utilidad el modelar un terreno mediante la interpolación de puntos de datos (Bernabe et al., 2016), la planeación de caminos y la construcción de mallas de calidad para el análisis de elementos finitos, etc. (Torti, Danese and Leporati, 2015); todas estas aplicaciones se basan en la triangulación de Delaunay, con esta finalidad se propone la triangulación restringida de Delaunay en un sistema híbrido paralelizado CPU-GPU, escalable, con lo que se obtiene un mejor rendimiento que la mejor aplicación basada en CPU (Tung, 2009).

Las imágenes hiperespectrales son usualmente capturadas y almacenadas desde satélites o aviones para ser transmitidas a 
estaciones terrestres, sin embargo, la gran cantidad de datos que contienen estás imágenes hacen necesario aplicar métodos de compresión y registro (Ordonez, Arguello and Heras, 2017) para reducir el volumen de datos antes de transmitir a un segmento terrestre.

Un aplicación para comprimir la información de imágenes es presentada en (Santos et al., 2013), en la que se utiliza una GPU CUDA, logrando así una mejora de 15.41 en velocidad de procesamiento, comparado con solo usar CPU. Otro factor que ocurre con el uso de las imágenes hiperespectrales, es la tendencia de que los pixeles se mezclen, por lo que es importante contar con un procesamiento para des mezclarlos de manera rápida y eficiente, por lo que Sergio Bernabé, et al. comparan dos plataformas paralelas, la GPU CUDA GeForce GTX 580 y la NVidia Tesla C1060 para llevar a cabo este procedimiento (Bernabe et al., 2013); de igual manera en (Barberis et al., 2013) se propone otro algoritmo de análisis de componentes Vertex, para desmezclar datos hiperespectrales mediante una tarjeta NVidia Fermi y CUDA; comparando el algoritmo basado en GPU vs CPU, se notó que el uso de GPU's es de gran ayuda para mejorar tiempos de procesamiento en este tipo de procesos.

Otro punto importante en el manejo de imágenes hiperespectrales es la identificación de sub espacios, para reducir la dimensionalidad correcta, lo que produce ganancias en el rendimiento y eficiencia del algoritmo, en (Torti et al., 2014) se propone la paralelización de este algoritmo basado en GPU comparándolo con un procesador digital de señales (DSP), se resalta que la implementación en DSP tiene un buen balance entre desempeño y consumo de energía, sin embargo el uso de números flotantes de doble precisión, es restrictivo, a comparación de un GPU. En (Wu et al., 2014), (Images and Martel, 2017) y (Guerra et al., 2017), se proponen métodos para desmezclar este tipo de imágenes, en el primer trabajo mediante la factorización de matrices dispersas no negativas, en el segundo mediante una cadena de procesamiento, $\mathrm{y}$ en el tercer trabajo utilizando el algoritmo rápido paralelo para desmezclar linealmente imágenes hiperespectrales, haciendo uso de GPU, al utilizar un método paralelo se comprobó la exactitud de los algoritmos propuestos.

La ortorectificación es una de los procesamientos más importantes en tareas de teledetección, este proceso remueve los efectos de la perspectiva de la geometría de la cámara y así los resultados pueden concordar con la escala de un mapa; en (Lei et al., 2014) se explora el uso de una GPU en una red agrupada para acelerar esta tarea. Una máquina de aprendizaje extrema, es utilizada para la clasificación de imágenes hiperespectrales por primera vez en (Javier, Investigaci and Heras, 2015), sin embargo también se utilizó una GPU para aplicar técnicas de conjuntos (obteniendo mejoras de $5 x$ ), algoritmos de regularización espacial y un esquema de clasificación espectral espacial.

La clasificación e identificación (Wu et al., 2016) de imágenes hiperespectrales mediante una máquina de vector de soporte tiene un alto costo computacional, por lo que en (Tan et al., 2015), (Wu, Wang, Plaza, Li, et al., 2015) y (Wu, Wang, Plaza and Li, 2015), se propone el uso de una GPU para mejorar este costo, logrando obtener una mejora en los procesos de prueba para datos de 103 bandas de 81.9x, 130x para el segundo trabajo y $210 \mathrm{x}$ para el tercer trabajo. Otra aplicación a las imágenes hiperespectrales es la generación automática de objetivos, que utilizando una versión en paralelo del algoritmo en una NVidia Tesla K20 se logró una mejora de 416x y 320x para conjuntos de datos $(512 * 614 * 224$ de 224 bandas y $35 * 350 * 188$ de 188 bandas respectivamente) (Li, Huang and Zhao, 
2015). Liuyang Fang, et al. muestran sus resultados de su interfaz de paso de mensaje, de datos adquiridos por el satélite Chino ZY$3 \mathrm{~m}$, utilizando una arquitectura CUDA, multiprocesamiento abierto, sus resultados arrojan que se redujo a 86.1s el tiempo de ejecución (Fang et al., 2015).

La profundidad óptica del aerosol (AOD), es una propiedad óptica importante del aerosol, que está involucrado en otras aplicaciones como, corrección atmosférica de las características de superficie detectadas remotamente, el monitoreo de erupciones volcánicas o incendios forestales, la calidad del aire e incluso los cambios climáticos a partir de datos satelitales. Sin embargo el procesamiento que se utiliza en esta técnica es computacionalmente costosa por la gran cantidad de datos a procesar, por lo que en (Liu et al., 2015) se propone el uso de una implementación basada en GPU-CUDA, para acelerar este procesamiento; se obtuvo 68x de incremento de velocidad en el estudio realizado, comparado con un $7 \mathrm{x}$ del basado en un procesador multinúcleo, y un menor consumo de energía utilizando GPU (3.15kJ con un hilo y 38.39kJ con 16 hilos) vs CPU (58.09kJ).

Una arquitectura CPU-GPU es propuesta en (Abbate et al., 2016) y (Garcia-
Rial, Ubeda-Medina and Grajal, 2017), para el procesamiento de datos den imágenes de radar, el primer caso de apertura sintética y el segundo radares en la banda de $\mathrm{THz}$, mediante la implementación y paralelización del algoritmo Chirp Scaling, al igual que en (Topa, 2017) que se evalúa el uso de GPU para simulación de momentos en aplicaciones electromagnéticas utilizando antenas de trayectoria plana. La segmentación también es relevante en el procesamiento de imágenes multidimensionales, sin embargo la mayoría de los algoritmos reducen la dimensión de las imágenes, con lo que se pierde información relevante en este proceso; en (Lopez-Fandino et al., 2017) se menciona la segmentación mediante GPU basado en una autómata celular, obteniendo mejor exactitud para cubiertas de tierra que otras técnicas de clasificación espacial.

La reconexión de líneas de contorno son elementos gráficos que permiten la caracterización 3D de terrenos en mapas de 2D, por lo que en (Song et al., 2017) se expone un método efectivo basado en GPU, se logró in mejoramiento de velocidad de $1360 \mathrm{x}$ con resultados idénticos a los obtenidos en CPU.

\section{Aplicaciones Médicas}

La velocidad de procesamiento para reconstrucción de imágenes de Tomografías por Emisión de Positrones (PET) han sido mejoradas en años recientes, mediante la descarga de trabajo a múltiples procesadores o GPU's, sin embargo el uso de clústeres de GPU no garantiza un incremento lineal en la velocidad de procesamiento, debido a la gran cantidad de datos que se necesitan transferir, por lo que en (Cui et al., 2013), se propone un algoritmo para obtener la expectativa de máxima verosimilitud de maximización para el uso de varios GPU's. En (Ha et al., 2013) se expone un método espacialmente simétrico, y variante de cambio que puede manejar de manera eficiente cualquier sistema de núcleo genérico la reconstrucción PET para imágenes tridimensionales (3D).

El registro de imágenes, es una técnica para definir la relación geométrica entre cada punto de dos imágenes, esto es de gran ayuda en las cirugías ayudadas por computadora, pero es indispensable que el tiempo de procesamiento de un valor 
pequeño, por lo que Kei Ikeda y Fumihiko Ino proponen un método eficiente para acelerar el registro no rígido de información mutua con CUDA (Ikeda, Ino and Hagihara, 2014).Otro tipo de imágenes que es necesario analizar son las visualizaciones tridimensionales de vasos cerebrales, que sirven para diagnosticar enfermedades, sin embargo, cuando se analizan varios vasos cerebrales es difícil decidir el orden de profundidad de manera clara; como solución en (Luo, 2013), (Won et al., 2013), se plantea la combinación de colores a distancia y la mejora de la profundidad estereoscópica se combinan con la reproducción de volúmenes pre integrados, basada en CUDA y su función avanzada de transferencia para una mejor percepción de la profundidad. En (Idzenga et al., 2014) se mencionan las ventajas de utilizar una GPU en la imagenología de ultrasonidos de dos dimensiones, mientras que en (Xanthis et al., 2014), se presenta un simulador (228 veces más rápido que la implementación serial basada en CPU) de imagenología de resonancia magnética (MRI), con ayuda de un ambiente basado en GPU.

Al utilizar una iluminación armónica esférica se pudo visualizar la microestructura cerebral en campos espacio angulares de alta fidelidad en imágenes MRI (Bista et al., 2014). La corrección de dispersión es de gran relevancia en las imágenes PET, para mejorar la exactitud de la estimación de dispersión se propuso simular múltiples contribuciones de dispersión en (Kim et al., 2014), basado en simulaciones Monte Carlo, considerando la migración de fotones e interacciones debido a la absorción fotoeléctrica y dispersión Compton, esto se realizó mediante un sistema híbrido CPUGPU y se obtuvo $128.3 x$ incremento de velocidad en este tipo de simulaciones; mientras que en (Wang, Li and Abubakar, 2015) se presenta un algoritmo de inversión de fuente de contraste con la misma finalidad.
En la actualidad la electrocardiografía (ECG) tiene una alta demanda debido al incremento de trastornos del ritmo cardiaco, sin embargo este tipo de estudios con frecuencia sufren de ruido relevante, por lo que es necesario el uso de filtros que usen wavelet, respuesta infinito y finito al impulso unitario, en (Niederhauser et al., 2015) se logró procesar el filtro wavelet 500 veces más rápido con el procesamiento en GPU. El uso de la tecnología de microarreglos de DNA (cDNA) (Katsigiannis, Zacharia and Maroulis, 2017), es una herramienta que ayuda a estudiar el nivel de expresión de miles de genes, la segmentación de imágenes de estos microarreglos es una de las principales etapas de este tipo de experimentos, sin embargo es un proceso arduo y desafiante debido a la mala calidad de las imágenes; Stamos Katsigiannis, et al. proponen un nuevo enfoque para este tipo de segmentación de DNA mediante el uso de una GPU (Katsigiannis, Zacharia and Maroulis, 2015).

La velocimetría es una técnica utilizada para medir la velocidad instantánea de fluidos y puede ser utilizada en el diseño de corazones artificiales, en (Moore, Leeser and King, 2015) se propone esta técnica mediante la especialización de núcleos en GPU y se pudo mejorar la velocidad de procesamiento de trabajos previos. Los métodos de reconstrucción iterativa en Tomografía Computarizada (CT) mediante rayos $\mathrm{X}$ (Projection et al., 2018), pueden proporcionar mejor calidad de imágenes que métodos analíticos (Fortmeier et al., 2016), pero su aplicación en la práctica son aún limitados debido al costo computacional en operaciones repetidas de proyección y retroproyección, sin embargo con el uso de GPU's es posible utilizar un método de trazado de rayos, para realizar este procedimiento, como se propone en (Nguyen and Lee, 2015). 
La imagenología ultrasónica es uno de los métodos de visualización más populares para examinar el interior de objetos opacos, en (Romero-Laorden et al., 2016) y (Chen, Chen and Huang, 2016) se realiza el análisis de estrategias de cómputo paralelo para acelerar el procesamiento de imagenología de ultrasonido mediante una GPU (Romero-Laorden et al., 2016). La delineación del ventrículo derecho cardíaco es esencial para generar mediciones clínicas, como la fracción de eyección y el volumen sistólico; en (Punithakumar, Boulanger and Noga, 2017) utilizan imágenes MRI para este procedimiento y una plataforma GPU, se observa que se pudo mejorar $19 x$ la velocidad de este tipo de procedimiento.

\section{Conclusiones}

Se observa que la utilidad de las GPU de NVIDIA, ha estado incrementándose en diferentes aplicaciones científicas y de Ingeniería, destacando su uso en aplicaciones geográficas con 25 aportaciones, 19 contribuciones en el área médica, 16 en el ámbito de video vigilancia y 8 para reconstrucción de imágenes. Y muchas más en congresos con estas mismas aplicaciones.

A pesar de que existen diferentes trabajos de revisión como el de Philippe Després y Xun Jia que se enfocaron a los desarrollos de reconstrucción de imágenes médicas basadas en GPU en 2017, desde una perspectiva de CT, PET, SPECT, MRI y US. Presentan las estrategias y los enfoques para aprovechar al máximo las GPU en la reconstrucción de imágenes, así como las aplicaciones innovadoras que surgen de una mayor capacidad informática. De igual manera analizan el futuro de la reconstrucción de imágenes basada en GPU, sustentado en las tendencias actuales de la computación de alto rendimiento (Després and Jia, 2017). El de Xun Jia et al. que presentan en su trabajo una revisión de las aplicaciones actuales de la GPU en los principales problemas relacionados con imagenología y los problemas relacionados con la radioterapia.

Además, se realizan una comparación en la solución de este tipo de problemática utilizando GPU y otras plataformas (Jia, Ziegenhein and Jiang, 2014). Esta revisión presenta los trabajos sobre procesamiento acelerado de imágenes médicas basado en GPU. La revisión abarca la aceleración GPU de operaciones básicas de procesamiento de imágenes (filtrado, interpolación, estimación de histogramas y transformaciones de distancia), los algoritmos más comúnmente utilizados en imágenes médicas (registro de imágenes, segmentación de imágenes y eliminación de imágenes) y algoritmos que son específicos de modalidades individuales (CT , PET, SPECT, MRI, fMRI, DTI, ultrasonido, imagen óptica y microscopía) (Eklund et al., 2013). Y en 2014 Balsa Rodríguez et al. realizaron un reporte donde examinan los enfoques de representación de volumen de la GPU, cubriendo diseño de red de muestreo, modelos de representación compactos, técnicas de compresión, arquitecturas de renderizado de GPU y técnicas rápidas de decodificación (Balsa Rodríguez et al., 2014).

No se había propuesto uno que abarcara diferentes aplicaciones de la GPU en diversas áreas de investigación. Este trabajo abarcó la utilidad de GPU's para acelerar la velocidad de procesamiento en áreas de la medicina, uso geográfico, reconstrucción de imágenes y video vigilancia. 


\section{Referencias}

Abbate, S. et al. 2016 'Extended Chirp Scaling on GPGPU’, IEEE Latin America Transactions, 14(6), pp. 2638-2643. doi: 10.1109/TLA.2016.7555231.

Alexiadis, D. S., Zarpalas, D. and Daras, P. 2013 'Real-Time , Full 3-D Reconstruction of Consumer Depth Cameras', IEEE Transactions on Multimedia, 15(2), pp. 339-358.

Bahri, H. et al. 2017 'Image feature extraction algorithm based on CUDA architecture: case study GFD and GCFD', IET Computers \& Digital Techniques, 11(4), pp. 125-132. doi: 10.1049/iet-cdt.2016.0135.

Balsa Rodríguez, M. et al. 2014 'State-of-the-art in compressed GPU-based direct volume rendering', Computer Graphics Forum, 33(6), pp. 77-100. doi: 10.1111/cgf.12280.

Barberis, A. et al. 2013 'Real-time implementation of the vertex component analysis algorithm on GPUs', IEEE Geoscience and Remote Sensing Letters, 10(2), pp. 251-255. doi: 10.1109/LGRS.2012.2200452.

Bernabe, S. et al. 2013 'Hyperspectral unmixing on GPUs and multi-core processors: A comparison', IEEE Journal of Selected Topics in Applied Earth Observations and Remote Sensing, 6(3), pp. 1386-1398. doi: 10.1109/JSTARS.2013.2254470.

Bernabe, S. et al. 2016 'Parallel Hyperspectral Coded Aperture for Compressive Sensing on GPUs', IEEE Journal of Selected Topics in Applied Earth Observations and Remote Sensing, 9(2), pp. 932-944. doi: 10.1109/JSTARS.2015.2436440.

Bista, S. et al. 2014 'Visualization of brain microstructure through spherical harmonics illumination of high fidelity spatio-angular fields', IEEE Transactions on Visualization and Computer Graphics, 20(12), pp. 25162525. doi: 10.1109/TVCG.2014.2346411.

Chen, Z., Chen, Y. and Huang, Q. 2016 'Development of a Wireless and Near Real-Time 3D Ultrasound Strain Imaging System', IEEE Transactions on Biomedical Circuits and Systems, 10(2), pp. 394-403. doi: 10.1109/TBCAS.2015.2420117.

Cui, J. et al. 2013 'Distributed MLEM: an iterative tomographic image reconstruction algorithm for distributed memory architectures.', IEEE Trans Med Imaging, 32(5), pp. 957-967. doi:

10.1109/TMI.2013.2252913.

Deligiannidis, L. and Arabnia, H. R. 2014 'Parallel Video Processing Techniques for Surveillance Applications', 2014 International Conference on Computational Science and Computational Intelligence, pp. 183-189. doi: 10.1109/CSCI.2014.38.

Després, P. and Jia, X. 2017 'A review of GPUbased medical image reconstruction’, Physica Medica, 42, pp. 76-92. doi: 10.1016/j.ejmp.2017.07.024.
Devani, U., Nikam, V. B. and Meshram, B. B. 2015 'Super-fast parallel eigenface implementation on GPU for face recognition', Proceedings of 2014 3rd International Conference on Parallel, Distributed and Grid Computing, PDGC 2014, pp. 130-136. doi: 10.1109/PDGC.2014.7030729.

Duchateau, J. et al. 2017 'An Out-of-Core Method for Physical Simulations on a Multi-GPU Architecture Using Lattice Boltzmann Method', Proceedings - 13th IEEE International Conference on Ubiquitous Intelligence and Computing, 13th IEEE International Conference on Advanced and Trusted Computing, 16th IEEE International Conference on Scalable Computing and Communications, IEEE Internationa, 581. doi: 10.1109/UIC-ATC-ScalComCBDCom-IoP-SmartWorld.2016.0099.

Eklund, A. et al. 2013 'Medical image processing on the GPU - Past, present and future', Medical Image Analysis. Elsevier B.V., 17(8), pp. 1073-1094. doi: 10.1016/j.media.2013.05.008.

Enfedaque, P., Auli-Llinas, F. and Moure, J. C. 2015 'Implementation of the DWT in a GPU through a Register-based Strategy', IEEE Transactions on Parallel and Distributed Systems, 26(12), pp. 3394-3406. doi: 10.1109/TPDS.2014.2384047.

Enfedaque, P., Auli-Llinas, F. and Moure, J. C. 2017 'GPU Implementation of Bitplane Coding with Parallel Coefficient Processing for High Performance Image Compression', IEEE Transactions on Parallel and Distributed Systems, 28(8), pp. 2272-2284. doi: 10.1109/TPDS.2017.2657506.

Fang, L. et al. 2015 'MOC-based parallel preprocessing of ZY-3 satellite images', IEEE Geoscience and Remote Sensing Letters, 12(2), pp. 419-423. doi: 10.1109/LGRS.2014.2345419.

Fatica, M. and Phillips, E. 2014 'Synthetic aperture radar imaging on a CUDA-enabled mobile platform', in 2014 IEEE High Performance Extreme Computing Conference (HPEC). IEEE, pp. 1-5. doi: 10.1109/HPEC.2014.7040960.

Feng, C., Zhang, X. and Gao, Z. 2015 ‘An Improved Image Super Resolution and Its Parallel ImplementationBased on CUDA', The Tenth International Conference on Digital Information Management, (Icdim), pp. 182-187.

Fortmeier, D. et al. 2016 'A virtual reality system for PTCD simulation using direct visuo-haptic rendering of partially segmented image data', IEEE Journal of Biomedical and Health Informatics, 20(1), pp. 355-366. doi: 10.1109/JBHI.2014.2381772.

Garcia-Rial, F., Ubeda-Medina, L. and Grajal, J. 2017 'Real-time GPU-based image processing for a 3-D THz radar', IEEE Transactions on Parallel and Distributed 
Systems, 28(10), pp. 2953-2964. doi:

10.1109/TPDS.2017.2687927.

González-Albo, B. and Bordons, M. 2011

'Articles vs. proceedings papers: Do they differ in research relevance and impact? A case study in the Library and Information Science field', Journal of Informetrics, 5(3), pp. 369-381. doi: 10.1016/j.joi.2011.01.011.

Green, O. 2018 'Efficient scalable median filtering using histogram-based operations', IEEE Transactions on Image Processing, 27(5), pp. 2217-2228. doi: 10.1109/TIP.2017.2781375.

Grosset, A. V. P. et al. 2017 'TOD-tree: Taskoverlapped direct send tree image compositing for hybrid MPI parallelism and GPUs', IEEE Transactions on Visualization and Computer Graphics, 23(6), pp. 16771680. doi: 10.1109/TVCG.2016.2542069.

Guerra, R. et al. 2017 'On the Evaluation of Different High-Performance Computing Platforms for Hyperspectral Imaging: An OpenCL-Based Approach', IEEE Journal of Selected Topics in Applied Earth Observations and Remote Sensing, 10(11), pp. 4879-4897. doi: 10.1109/JSTARS.2017.2737958.

Guo, X. et al. 2016 'Parallel Computation of Aerial Target Reflection of Background Infrared Radiation: Performance Comparison of OpenMP, OpenACC, and CUDA Implementations', IEEE Journal of Selected Topics in Applied Earth Observations and Remote Sensing, 9(4), pp. 1653-1662. doi: 10.1109/JSTARS.2016.2516503.

Gutierrez, P. D. et al. 2014 'A high performance fingerprint matching system for large databases based on GPU', IEEE Transactions on Information Forensics and Security, 9(1), pp. 62-71. doi: 10.1109/TIFS.2013.2291220.

Ha, S. et al. 2013 'GPU-accelerated forward and back-projections with spatially varying kernels for 3D DIRECT TOF PET reconstruction', IEEE Transactions on Nuclear Science, 60(1), pp. 166-173. doi: 10.1109/TNS.2012.2233754.

Haythem, B. et al. 2013 'Contribution to the implementation of computer vision application on a GPU', 2013 International Conference on Control, Decision and Information Technologies, CoDIT 2013, pp. 319-324. doi: 10.1109/CoDIT.2013.6689564.

Haythem, B. et al. 2014 'Fast Generalized Fourier Descriptor for object recognition of image using CUDA', 2014 World Symposium on Computer Applications and Research, WSCAR 2014, (4). doi: 10.1109/WSCAR.2014.6916817.

Heidari, H. 2013 'Accelerating of Color Moments and Texture Features Extraction Using GPU Based Parallel Computing', 8th Iranian Conference on Machine Vision and Image Processing MVIP, pp. 430-435. doi: 10.1109/IranianMVIP.2013.6780024.

Idzenga, T. et al. (2014) 'Fast 2-D ultrasound strain imaging: The benefits of using a GPU', IEEE Transactions on Ultrasonics, Ferroelectrics, and Frequency Control, 61(1), pp. 207-213. doi:

\subsection{9/TUFFC.2014.6689790.}

Ikeda, K., Ino, F. and Hagihara, K. 2014

'Efficient acceleration of mutual information computation for nonrigid registration using CUDA', IEEE Journal of Biomedical and Health Informatics, 18(3), pp. 956-968. doi: 10.1109/JBHI.2014.2310745.

Images, U. H. and Martel, E. 2017 'A GPU-Based Processing Chain for Linearly’, 10(3), pp. 818-834.

Javier, L., Investigaci, C. S. De and Heras, D. B. 2015 'Efficient Classification of Hyperspectral Images on Commodity GPUs using ELM-based Techniques’, 8(6), pp. 2884-2893.

Jha, S. and Trivedi, P. 2013 'An automated video surveillance system using Viewpoint Feature Histogram and CUDA-enabled GPUs', Proceedings of the 2013 International Conference on Advances in Computing, Communications and Informatics, ICACCI 2013, pp. 18121816. doi: 10.1109/ICACCI.2013.6637456.

Jia, X., Ziegenhein, P. and Jiang, S. B. 2014 'GPU-based high-performance computing for radiation therapy’, Physics in Medicine and Biology, 59(4). doi: 10.1088/0031-9155/59/4/R151.

Jiansen Li et al. 2014 'Accelerating the reconstruction of magnetic resonance imaging by threedimensional dual-dictionary learning using CUDA', in 2014 36th Annual International Conference of the IEEE Engineering in Medicine and Biology Society. IEEE, pp. 2412-2415. doi: 10.1109/EMBC.2014.6944108.

Katsigiannis, S., Zacharia, E. and Maroulis, D. 2015 'Grow-Cut Based Automatic cDNA Microarray Image Segmentation', IEEE Trans Nanobioscience, 14(1), pp. 138-145. doi: 10.1109/tnb.2014.2369961 LB Katsigiannis2015.

Katsigiannis, S., Zacharia, E. and Maroulis, Di. 2017 'MIGS-GPU: Microarray Image Gridding and Segmentation on the GPU', IEEE Journal of Biomedical and Health Informatics, 21(3), pp. 867-874. doi: 10.1109/JBHI.2016.2537922.

Kau, L. J. and Chen, C. S. 2013 'Speeding up the runtime performance for lossless image coding on GPUs with CUDA', Proceedings - IEEE International Symposium on Circuits and Systems, (1), pp. 2868-2871. doi: 10.1109/ISCAS.2013.6572477.

Kim, K. S. et al. 2014 'Ultra-fast hybrid CPUGPU multiple scatter simulation for 3-D PET', IEEE Journal of Biomedical and Health Informatics, 18(1), pp. 148-156. doi: 10.1109/JBHI.2013.2267016.

Kowalczuk, J., Psota, E. T. and Perez, L. C. 2013 'Real-Time Stereo Matching on \{CUDA \} Using an Iterative Refinement Method for Adaptive Support Weight Correspondences’, IEEE Trans. CSVT, 23(1), pp. 94-104.

Lee, I.-H. et al. 2013 'Accelerating motioncompensated adaptive color Doppler engine on CUDAbased GPU platform', Signal Processing Systems (SiPS), 2013 IEEE Workshop on, pp. 1-6. 
Lei, Z. et al. 2014 'Stream model-based orthorectification in a GPU cluster environment', IEEE Geoscience and Remote Sensing Letters, 11(12), pp. 21152119. doi: 10.1109/LGRS.2014.2320991.

Li, X., Huang, B. and Zhao, K. 2015 'Massively Parallel GPU Design of Automatic Target Generation Process in Hyperspectral Imagery', IEEE Journal of Selected Topics in Applied Earth Observations and Remote Sensing, 8(6), pp. 2862-2869. doi: 10.1109/JSTARS.2014.2347299.

Li, Y., Mogelmose, A. and Trivedi, M. M. 2016 'Pushing the "Speed Limit": High-Accuracy US Traffic Sign Recognition With Convolutional Neural Networks', IEEE Transactions on Intelligent Vehicles, 1(2), pp. 167176. doi: 10.1109/TIV.2016.2615523.

Liu, J. et al. 2015 'Multicore Processors and Graphics Processing Unit Accelerators for Parallel Retrieval of Aerosol Optical Depth From Satellite Data: Implementation, Performance, and Energy Efficiency', IEEE Journal of Selected Topics in Applied Earth Observations and Remote Sensing, 8(5), pp. 2306-2317. doi: 10.1109/JSTARS.2015.2438893.

Loock, W. Van et al. 2014 'Short Papers', 35(12), pp. 1-6.

Lopez-Fandino, J. et al. 2017 'GPU Projection of ECAS-II Segmenter for Hyperspectral Images Based on Cellular Automata', IEEE Journal of Selected Topics in Applied Earth Observations and Remote Sensing, 10(1), pp. 20-28. doi: 10.1109/JSTARS.2016.2588530.

Luo, Y. L. 2013 'Effectively visualizing the spatial structure of cerebral blood vessels', Computing in Science and Engineering, 15(2), pp. 41-46. doi: 10.1109/MCSE.2013.25.

Marcellino, L. and Navarra, G. 2016 'A GPUaccelerated SVD algorithm, based on QR factorization and Givens Rotations , for DWI denoising .' doi: 10.1109/SITIS.2016.117.

Method, A. I. D. et al. 2014 'GPU-Parallel Implementation of the Edge-Directed’, 10(9), pp. 746-753.

Moore, G. E. 1998 'Cramming more components onto integrated circuits', Proceedings of the IEEE, 86(1), pp. 82-85. doi: 10.1109/JPROC.1998.658762.

Moore, G. E. 2005 'Excerpts from A Conversation with Gordon Moore: Moore’s Law’, Intel, pp. 1-2. Available at:

http://large.stanford.edu/courses/2012/ph250/lee1/docs/Exc epts_A_Conversation_with_Gordon_Moore.pdf\%0Ahttp:// download.intel.com/museum/Moores_law/Videotranscripts/excepts_a_Conversation_with_gordon_Moore.p df.

Moore, N., Leeser, M. and King, L. S. 2015 'Kernel specialization provides adaptable GPU code for particle image velocimetry', IEEE Transactions on Parallel and Distributed Systems, 26(4), pp. 1049-1058. doi: 10.1109/TPDS.2014.2317721.
Nguyen, V. G. and Lee, S. J. 2015 'Parallelizing a matched pair of ray-tracing projector and backprojector for iterative cone-beam CT reconstruction', IEEE Transactions on Nuclear Science, 62(1), pp. 171-181. doi: 10.1109/TNS.2015.2388553.

Niederhauser, T. et al. 2015 'Graphics-processorunit-based parallelization of optimized baseline wander filtering algorithms for long-term electrocardiography', IEEE Transactions on Biomedical Engineering, 62(6), pp. 1576-1584. doi: 10.1109/TBME.2015.2395456.

NVIDIA no date Procesamiento paralelo CUDA | Qué es CUDA | NVIDIA. Available at:

https://developer.nvidia.com/cuda-zone.

Nykl, S., Mourning, C. and Chelberg, D. 2014 'Interactive Mesostructures withVolumetric Collisions', IEEE Transactions on Visualization and Computer Graphics, 20(7), pp. 970-982. doi: 10.1109/TVCG.2014.2317700.

Optimization, G. 2018 'Image Autoregressive Interpolation Model Using’, 14(2), pp. 426-436.

Ordonez, A., Arguello, F. and Heras, D. B. 2017 'GPU accelerated FFT-based registration of hyperspectral scenes', IEEE Journal of Selected Topics in Applied Earth Observations and Remote Sensing, 10(11), pp. 4869-4878. doi: 10.1109/JSTARS.2017.2734052.

Pawar, D. 2017 'GPU Based Background Subtraction Using CUDA : State of the Art', pp. 12011204.

Phusomsai, W. and So-in, C. 2016 'Brain Tumor Cell Recognition Schemes using Image Processing with Parallel ELM Classifications on GPU'.

Projection, B. et al. 2018 'A Look-Up TableBased Ray Integration’, 37(2), pp. 361-371.

Punithakumar, K., Boulanger, P. and Noga, M. 2017 'A GPU-Accelerated Deformable Image Registration Algorithm with Applications to Right Ventricular Segmentation', IEEE Access, 5, pp. 20374-20382. doi: 10.1109/ACCESS.2017.2755863.

Rakvic, R., Broussard, R. and Ngo, H. A. U. 2016 'Energy Efficient Iris Recognition With Graphics Processing Units', 4, pp. 2831-2839. doi: 10.1109/ACCESS.2016.2571747.

Romero-Laorden, D. et al. 2016 'Analysis of Parallel Computing Strategies to Accelerate Ultrasound Imaging Processes', IEEE Transactions on Parallel and Distributed Systems, 27(12), pp. 3429-3440. doi: 10.1109/TPDS.2016.2544312.

Santos, L. et al. 2013 'Highly-parallel gpu architecture for lossy hyperspectral image compression', IEEE Journal of Selected Topics in Applied Earth Observations and Remote Sensing, 6(2), pp. 670-681. doi: 10.1109/JSTARS.2013.2247975.

Saxena, S., Sharma, S. and Sharma, N. 2014 'Image registration techniques using parallel computing in multicore environment and its applications in medical 
imaging: An overview’, 2014 International Conference on Computer and Communication Technology (ICCCT), pp. 97-104. doi: 10.1109/ICCCT.2014.7001475.

Shuai, L. et al. 2017 'Motion Capture with Ellipsoidal Skeleton Using Multiple Depth Cameras', IEEE Transactions on Visualization and Computer Graphics, 23(2), pp. 1085-1098. doi: 10.1109/TVCG.2016.2520926.

Song, J. et al. 2017 'The Reconnection of Contour Lines from Scanned Color Images of Topographical Maps Based on GPU Implementation', 10(2), pp. 400-408.

Sun, L. C. et al. 2013 'Acceleration algorithm for CUDA-based face detection', 2013 IEEE International Conference on Signal Processing, Communications and Computing, ICSPCC 2013. doi: 10.1109/ICSPCC.2013.6664139.

Sun, X. and Wang, R. 2015 'Fast Smoke Detection for video surveillance Using CUDA', 14(2), pp. 725-733. doi: 10.1109/BigMM.2015.86.

Tagliavini, G., Cesarini, D. and Marongiu, A. 2018 'Unleashing Fine-Grained Parallelism on Embedded Many-Core Accelerators with Lightweight OpenMP Tasking', IEEE Transactions on Parallel and Distributed Systems, 9219(X), pp. 1-1. doi: 10.1109/TPDS.2018.2814602.

Tan, K. et al. 2015 'GPU Parallel Implementation of Support Vector Machines for Hyperspectral Image Classification', IEEE Journal of Selected Topics in Applied Earth Observations and Remote Sensing, 8(10), pp. 46474656. doi: 10.1109/JSTARS.2015.2453411.

Topa, T. 2017 'Load-Balanced Fortran-Based Out-of-GPU Memory Implementation of the Method of Moments', IEEE Antennas and Wireless Propagation Letters, 16, pp. 813-816. doi: 10.1109/LAWP.2016.2605042.

Torti, E. et al. 2014 'Real-Time Identi fi cation of Hyperspectral Subspaces’, 7(6), pp. 2680-2687.

Torti, E., Danese, G. and Leporati, F. 2015 'A Hybrid CPU - GPU Real-Time Hyperspectral’, 9(2), pp. 17.

Tung, C. T. 2009 'Computing 2D Delaunay Triangulation using GPU’, Comp.Nus.Edu.Sg, 19(5), pp. 736-748. Available at: http://www.comp.nus.edu.sg/ tants/delaunay2DDownload_ files/cao_hyp_2009.pdf.

Vogel, T. 2013 'All the way to CUDA', Computing in Science and Engineering, 15(5), pp. 6-8. doi: 10.1109/MCSE.2013.101.

Vokorokos, L. et al. 2014 'Increasing efficiency of the sequential algorithms programs execution using CUDA', SAMI 2014 - IEEE 12th International Symposium on Applied Machine Intelligence and Informatics, Proceedings, pp. 281-284. doi:

10.1109/SAMI.2014.6822422.

Wang, X. Y., Li, M. and Abubakar, A. 2015 'Acceleration of multiplicative regularized contrast source inversion algorithm using paralleled computing device', Microwave Conference (APMC), 2015 Asia-Pacific, 3(2), pp. 1-3.

Weinlich, A. et al. 2013 'Volumetric deformation compensation in CUDA for coding of dynamic cardiac images', in 2013 Picture Coding Symposium (PCS). IEEE, pp. 189-192. doi: 10.1109/PCS.2013.6737715.

Won, J. H. et al. 2013 'Uncluttered single-image visualization of vascular structures using GPU and integer programming', IEEE Transactions on Visualization and Computer Graphics, 19(1), pp. 81-93. doi: 10.1109/TVCG.2012.25.

Wu, X. et al. 2016 'GPU-Based Parallel Design of the Hyperspectral Signal Subspace Identification by Minimum Error (HySime)', IEEE Journal of Selected Topics in Applied Earth Observations and Remote Sensing, 9(9), pp. 4400-4406. doi: 10.1109/JSTARS.2016.2574876.

Wu, Z. et al. 2014 'Sparse non-negative matrix factorization on GPUs for hyperspectral unmixing', IEEE Journal of Selected Topics in Applied Earth Observations and Remote Sensing, 7(8), pp. 3640-3649. doi: 10.1109/JSTARS.2014.2315045.

Wu, Z., Wang, Q., Plaza, A., Li, J., et al. 2015 'Parallel Implementation of Sparse Representation Classifiers for Hyperspectral Imagery on GPUs', IEEE Journal of Selected Topics in Applied Earth Observations and Remote Sensing, 8(6), pp. 2912-2925. doi: 10.1109/JSTARS.2015.2413831.

Wu, Z., Wang, Q., Plaza, A. and Li, J. 2015 'Parallel Spatial - Spectral Hyperspectral Image Classification With Sparse Representation and Markov Random Fields on GPUs', IEEE Journal of Selected Topics in Applied Earth Observations and Remote Sensing, 8(6), pp. 2926-2938. doi: 10.1109/JSTARS.2015.2413931.

Xanthis, C. G. et al. 2014 'MRISIMUL: A GPUbased parallel approach to MRI simulations', IEEE Transactions on Medical Imaging, 33(3), pp. 607-617. doi: 10.1109/TMI.2013.2292119.

Xu, F., Dinavahi, V. and Xu, X. 2016 'Parallel Computation of Wrench Model for Commutated Magnetically Levitated Planar Actuator', IEEE Transactions on Industrial Electronics, 63(12), pp. 76217631. doi: 10.1109/TIE.2016.2592866.

Yazdanpanah, A. P. et al. 2014 'A CUDA based implementation of locally-and feature-adaptive diffusion based image denoising algorithm', ITNG 2014 -

Proceedings of the 11th International Conference on Information Technology: New Generations, pp. 388-393. doi: 10.1109/ITNG.2014.113.

Yu, D. et al. 2015 'Fast Rotation-Free Feature Based Image Registration Using Improved N-SIFT and GMM Based Parallel Optimization', IEEE Transactions on Biomedical Engineering, 9294(c), pp. 1-1. doi: 10.1109/TBME.2015.2465855.

Zhang, Z. et al. 2013 'GPU-Accelerated RealTime Tracking of Full-Body Motion With Multi-Layer 
Search', IEEE Transactions on Multimedia, 15(1), pp. 106119. doi: 10.1109/TMM.2012.2225040.

Zhao, M. et al. 2015 'Real-time and temporalcoherent foreground extraction with commodity RGBD camera', IEEE Journal on Selected Topics in Signal Processing, 9(3), pp. 449-461. doi: 10.1109/JSTSP.2014.2382476.

Zhao, Z., Zhang, X. and Fang, Y. 2015 'Stacked multilayer self-organizing map for background modeling', IEEE Transactions on Image Processing, 24(9), pp. 28412850. doi: 10.1109/TIP.2015.2427519.
Zhou, X., Li, X. and Hu, W. 2016 'Learning A Superpixel-Driven Speed Function for Level Set Tracking', IEEE Transactions on Cybernetics, 46(7), pp. 1498-1510. doi: 10.1109/TCYB.2015.2451100.

Zhu, Z. et al. 2013 'Research on CUDA-based image parallel dense matching', Proceedings - 2013 Chinese Automation Congress, CAC 2013, pp. 482-486. doi: 10.1109/CAC.2013.6775782.

Zwan, M. Van Der, Codreanu, V. and Telea, A. 2016 'CUBu : Universal real-time bundling for large', 22(12), pp. 1-14. 